\title{
BMJ Open Relationship between life satisfaction and preventable hospitalisations: a population-based cohort study in Ontario, Canada
}

\author{
Eric De Prophetis, ${ }^{1}$ Vivek Goel, ${ }^{1,2}$ Tristan Watson, ${ }^{3}$ Laura C Rosella (1) ${ }^{1}$
}

To cite: De Prophetis E, Goel V, Watson T, et al. Relationship between life satisfaction and preventable hospitalisations: a population-based cohort study in Ontario, Canada. BMJ Open 2020;10:e032837. doi:10.1136/ bmjopen-2019-032837

- Prepublication history and additional material for this paper are available online. To view these files, please visit the journal online (http://dx.doi. org/10.1136/bmjopen-2019032837).

Received 08 August 2019 Revised 06 February 2020 Accepted 07 February 2020

Check for updates

(c) Author(s) (or their employer(s)) 2020. Re-use permitted under CC BY-NC. No commercial re-use. See rights and permissions. Published by BMJ.

1Dalla Lana School of Public Health, University of Toronto, Toronto, Ontario, Canada 'Institute of Health Policy, Management and Evaluation, University of Toronto, Toronto, Ontario, Canada

${ }^{3}$ Populations and Public Health, ICES, Toronto, Ontario, Canada

Correspondence to

Dr Laura C Rosella;

laura.rosella@utoronto.ca

\section{ABSTRACT}

Objective To examine if low life satisfaction is associated with an increased risk of being hospitalised for an ambulatory care sensitive condition (ACSC), in comparison to high life satisfaction

Design and setting Population-based cohort study of adults from Ontario, Canada. Baseline data were captured through the Canadian Community Health Survey (CCHS) and linked to health administrative data for follow-up information.

Participants 129467 men and women between the ages 18 and 74.

Main outcome measures Time to avoidable hospitalisations defined by ACSCs.

Results Life satisfaction was measured at baseline through the CCHS and follow-up information on ACSC hospitalisations were captured by linking participant respondents to hospitalisation records covered under a single payer health system. Within the study time frame (maximum of 14 years), 3037 individuals were hospitalised. Older men in the lowest household income quintile were more likely to be hospitalised with an ACSC. After controlling for age, sex, socioeconomic status (SES) and other behavioural factors, low life satisfaction at baseline had a strong relationship with future hospitalisations for ACSCs (HR 2.71; 95\% Cl 1.87 to 3.93). The hazards were highest for those who jointly had the lowest levels of life satisfaction and low household income (HR 3.80; 95\% Cl 2.13 to 6.73). Results did not meaningful change after running a competing risk survival analysis.

Conclusions This study demonstrates that poor life satisfaction is associated with hospitalisations for ACSCs after adjustment for several confounders. Furthermore, the magnitude of this relationship was greater for those who were more socioeconomically disadvantaged. This study adds to the existing literature on the impact of life satisfaction on health system outcomes by documenting its impact on avoidable hospitalisations in a universal health system.

\section{INTRODUCTION}

There has been broad recognition that upstream determinants have an influence on a range of health outcomes, including social determinants and risky health behaviours. In addition, subjective well-being, specifically life

\section{Strengths and limitations of this study}

- This is the largest study to combine detailed survey variables linked to complete hospitalisation outcomes form from health administrative databases to study the impact of life satisfaction on ambulatory care sensitive conditions (ACSCs).

- The linkage of various databases allowed for control of a wide range of confounders in addition to objective measures of comorbidity.

- The linkage and study design allowed measurement of baseline life satisfaction on a sample of individuals who did not have an ACSCs in the 2 years prior, which overcomes a limitation of previous studies that may have been influenced by the bidirectional relationship between poor health and life satisfaction.

- Life satisfaction was measured at one point in time, and thus, we were unable to capture changes in life satisfaction over the study period.

- This study did not directly address mechanisms by which life satisfaction could influence ACSCs.

satisfaction is increasingly being recognised as playing an important role. ${ }^{1-3}$ Shifting from health outcomes to a health system perspective, many ambulatory care sensitive conditions (ACSCs) are preventable and result in high amounts of healthcare resources if left unmanaged. ${ }^{4} 5$ Hospitalisations for these conditions (eg, diabetes) are regarded as avoidable due to the relationship of timely access to primary care preventing complications and acute episodes. ${ }^{5-7}$ In many countries, hospitalisations for these conditions are used as an indicator to measure the effectiveness of the healthcare system. ${ }^{8}$

Positive affect (ie, positive emotions, happiness), is the degree to which an individual experiences positive emotion, ${ }^{9}$ has been shown to be independently associated with reduced risk of coronary heart disease, even after adjustment for depressive symptoms. ${ }^{10}$ Similarly, life satisfaction is associated with a reduced risk 


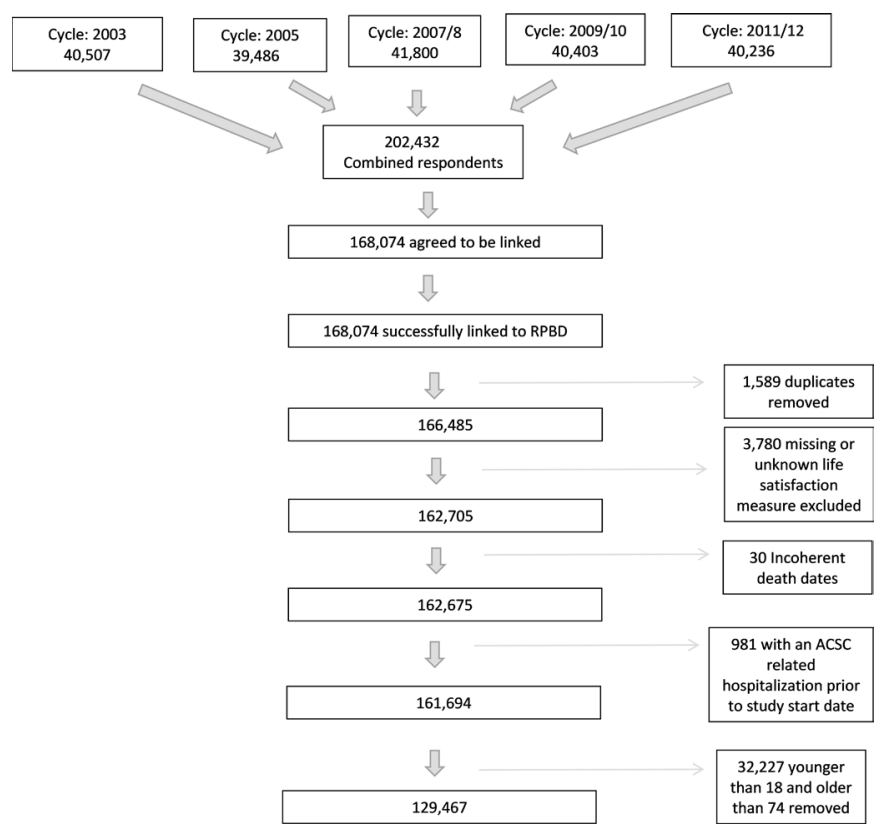

Figure 1 Flow chart of study participants. ACSC, ambulatory care sensitive condition; RPDB, Registered Persons Database.

of various chronic conditions. ${ }^{2}{ }^{11}$ Evidence from a recent prospective cohort study concluded lower life satisfaction was associated with a higher incidence of cancer, stroke and type 2 diabetes ${ }^{11}$ and others have shown its relationship with health behaviours. ${ }^{12}$ Positive affect is distinct from life satisfaction in that it is an emotional response describing positive emotions or happiness, which can be transitory. This is conceptually distinct from the more stable and complex measure of being satisfied with life, which is based on an individual's self-judgements of several factors that they feel are valuable to their life.

Research on life satisfaction has also recently shown to impact future high-health care use. ${ }^{13}$ Individuals with lower life satisfaction had three times the odds of being in the top $5 \%$ of healthcare resource utilisation. ${ }^{13}$ However, another important system indicator that is absent from the current life satisfaction literature is avoidable hospitalisations, such as those caused by ACSCs. Given the evidence around the interrelationships between emotional factors and chronic disease self -management ${ }^{14}$ and in particular how emotional and psychological distress can influence the ability of a patient to manage their chronic condition, ${ }^{15} 16$ more research is needed that quantitatively measures the impact of subjective well-being on important healthcare system outcomes.

On a recent search, only eight studies were identified that fit the criteria of a similar exposure to life satisfaction and hospitalisations for one or more ACSCs as an outcome. Previous studies mostly focused on singular conditions, with small sample sizes, limited follow-up time and clinical or convenience-based samples. ${ }^{1718}$ Regarding the length of follow-up, these ranged from 6 months ${ }^{19}$ to 4 years. ${ }^{20}$ Studies with shorter follow-up times can be limited because many of the conditions regarded as
ACSCs are also chronic diseases (eg, diabetes) require a longer follow-up time necessary to allow for outcomes to be observed. Furthermore, hospitalisations for ACSCs are a relatively rare event in Canada where people with an ACSC hospitalisation constitutes only $0.4 \%$ of the population aged $12-74,{ }^{21}$ and therefore, the limited sample sizes in previous studies may not be sufficient to observe an effect. We address these limitations by conducting the largest population-based cohort study to date. The low rate of ACSCs is typical of similar health systems in Europe, the UK and Australia.

The primary objective of this study was to determine if poor life satisfaction increased the risk of being hospitalised for an ACSC in a relatively healthy baseline cohort among an adult population using linked survey and complete hospitalisation records. Our secondary objective was to determine if this association was stronger among those with lower socioeconomic status (SES).

\section{METHODS \\ Participants}

The study was a longitudinal population-based cohort study of adult Ontario participants of the Canadian Community Health Survey (CCHS) pooled across five cycles: cycle 2.1 (2003-2004), cycle 3.1 (2005-2006), cycle (2007-2008), cycle (2009-2010) and cycle (2011-2012). The CCHS conducted surveys on a 2-year collection cycle (ie, 2003, 2005), therefore, no cycle existed for 2004 and 2006 and cycle naming conventions changed after 2005 to remove cycle numbers. The CCHS is a cross-sectional survey administered by Statistics Canada, representative of $98 \%$ of the Canadian population aged $\geq 12$ years living in private dwellings with response rates $>75 \% .^{22} 23$ The respondents in the CCHS survey consented to participate in the survey and have their data linked to administrative data for research purposes. Where the CCHS is a cross-sectional survey, the longitudinal aspect of this study is achieved by retrieving the outcome measure from hospital administrative databases. The data were linked to population-based health administrative data held at ICES. These data sources capture all hospitalisation records for every person living in the province of Ontario covered under the single-payer health system.

Eighty per cent of the CCHS survey respondents consented to have their data linked to the single-payer health insurance data, referred to as the Ontario Health Insurance Plan, which captures all related healthcare encounters. All survey respondents were linked to Ontario's population registry, the Registered Persons Database (RPDB), which captures core demographic and clinical information as well as death, in addition to the Discharge Abstract Database. The analytical sample included adults (aged 18-74) who reported on life satisfaction and did not have an ACSC-related hospitalisation in the 2 years before their CCHS interview date (figure 1). 


\section{Measures}

Self-reported life satisfaction, the primary exposure variable, is captured from CCHS. The question that respondents answered regarding life satisfaction was, 'How satisfied are you with your life in general?' With response options being: very satisfied, satisfied, neither satisfied nor dissatisfied, dissatisfied or very dissatisfied. Due to small sample sizes within each category, we collapsed the categories of dissatisfied and very dissatisfied. Furthermore, we did not hypothesise significant conceptual differences between these two categories related to hospitalisations for ACSCs.

The primary outcome variable was hospitalisations for an ACSC, which we used as a composite outcome. The ACSCs that we chose to report on are grand mal status and other epileptic convulsions, chronic obstructive pulmonary disease, asthma, diabetes, heart failure and pulmonary oedema, hypertension and angina. These conditions are in accordance with the Canadian Institute for Health Information's (CIHI) methodology ${ }^{24}$ and this composite outcome is an established health system indicator in Canada. The CIHI indicator applies only to individuals under the age of 75 as the hospitalisations in those above the age of 75 are not as clearly avoidable through timely and effective primary care. See online supplementary table 1 for the list of included conditions and their corresponding International Classification of Diseases 10th Revision (ICD-10) codes.

Aggregate diagnosis groups (ADGs) ${ }^{25}$ were captured through administrative data as a summary measure of comorbidity and are based on the Johns Hopkins ACG System, which is a person-focused, diagnosis-based method of categorising subjects' illnesses. ${ }^{22}$ ADGs have previously been used and validated as a reliable method of comorbidity adjustment in the Ontario population, ${ }^{26}$ and we used V.10.0.1 in this analysis. Additionally, we used the Ontario Marginalization Index (ON-Marg) as a measure of SES. The ON-Marg is a census-based, geographically derived index that was used to calculate area-level material deprivation. ${ }^{27}$ Specifically, the material deprivation dimension measures the proportion of the population within a geographical region that is low income, without high school diploma, lone parent families, receiving government transfer payments, unemployed and living in dwellings in need of repair. All other covariates were captured through self-report from the CCHS interview questions.

\section{CCHS variables}

Household income quintile categorises individuals based on their total household income in addition to the number of individuals living in the household. Individuals are then ranked from the lowest levels of household income (Q1) to the highest (Q5). Body mass index (BMI) was categorised into five categories ranging from 'underweight' (BMI $<18.5 \mathrm{~kg} / \mathrm{m}^{2}$ ) to 'severely obese' (BMI $\left.>34.9 \mathrm{~kg} / \mathrm{m}^{2}\right)$. Physical activity was based on an individual's self-reported daily energy expenditure and further categorised into three levels: active, moderately active and inactive. Smoking status measured an individuals' selfreported past and present smoking habits by considering both the total amount of cigarettes smoked and the type of smoker they are (eg, daily vs occasional). This variable was categorised into three levels: current smoker, former smoker and never smoker. Alcohol consumption was based on the participant's sex and the quantity of alcohol consumed each day. This variable was then categorised into four levels: heavy drinker, moderate drinker, light drinker and never drinker. Mood disease was captured through the CCHS interview question 'have you ever been diagnosed by a health professional for depression, bipolar disorder, mania or dysthymia?' This variable was used to control for depression. Anxiety disorder, which was captured through the question: 'have you ever been diagnosed by a health professional for an anxiety disorder such as a phobia, obsessive-compulsive disorder or panic disorder?' Education level is a derived variable which indicates the highest level of education acquired by the participant; this variable was explored as a potential indicator for SES.

\section{Statistical analysis}

We calculated the distribution of demographic, socioeconomic, health status and behaviour characteristics according to ACSCs and life satisfaction. Excluding those with an ACSC in the year prior allowed for the investigation of the upstream determinants (ie, the factors associated with future development of an ACSC hospitalisation in a cohort who were without a recent hospitalisation for one of these conditions).

Cox proportional hazards models were used to estimate the hazards associated with baseline life satisfaction on the risk of being hospitalised for an ACSC. Time is defined as survey date until disease or censoring for study endpoint (max follow-up until 31 March 2017) or death. The models were used to quantify the association between life satisfaction and the hazard of being hospitalised for an ACSC using 'very satisfied' as the referent category. We calculated unadjusted, age-adjusted and sexadjusted, minimally adjusted and fully adjusted models to transparently demonstrate the impact of adjustment. The minimally adjusted model controlled for age, sex and household income while the fully adjusted model included age, sex, household income, smoking status, alcohol consumption, physical activity and BMI. In order to show the fully adjusted results were not meaningfully affected by over comorbidity, mood disorders and anxiety, we ran three additional models, which controlled for ADG score, mood disease and anxiety separately. We ran these models separately in order to quantify their impact on the life satisfaction hazards in the fully adjusted model that controlled for sociodemographic and behavioural factors that we conceptualised as confounders (ie, age, sex, household income, smoking status, alcohol consumption, physical activity and BMI). 
Table 1 Weighted* distributions of baseline characteristics across five levels of life satisfaction $(n=129467)$

\begin{tabular}{|c|c|c|c|c|c|c|}
\hline & $\begin{array}{l}\text { Very } \\
\text { satisfied } \\
(n=49502)\end{array}$ & $\begin{array}{l}\text { Satisfied } \\
(\mathrm{n}=67978)\end{array}$ & $\begin{array}{l}\text { Neither satisfied } \\
\text { or dissatisfied } \\
\text { ( } n=7312)\end{array}$ & $\begin{array}{l}\text { Dissatisfied } \\
(\mathrm{n}=3779)\end{array}$ & $\begin{array}{l}\text { Very } \\
\text { dissatisfied } \\
(n=896)\end{array}$ & $\begin{array}{l}\text { Unweighted } N \\
(n=129467)\end{array}$ \\
\hline \multicolumn{7}{|l|}{ ACSC hospitalisation } \\
\hline Yes & 1.12 & 1.61 & 2.51 & 4.81 & 5.39 & 3037 \\
\hline No & 98.88 & 98.39 & 97.49 & 95.19 & 94.61 & 126430 \\
\hline \multicolumn{7}{|l|}{ Sex } \\
\hline Male & 47.5 & 50.53 & 47.34 & 49.93 & 47.32 & 59292 \\
\hline Female & 52.5 & 49.47 & 52.66 & 50.07 & 52.68 & 70175 \\
\hline \multicolumn{7}{|l|}{ Age group } \\
\hline 18-34 & 30.64 & 33.09 & 30.78 & 23.91 & 14.53 & 35255 \\
\hline $35-49$ & 31.9 & 33.21 & 31.14 & 36.12 & 33.6 & 35063 \\
\hline $50-64$ & 26.31 & 24.97 & 27.37 & 31.04 & 41.39 & 38662 \\
\hline $65-74$ & 11.15 & 8.72 & 10.7 & 8.93 & 10.49 & 20487 \\
\hline \multicolumn{7}{|l|}{$\mathrm{BMI}\left(\mathrm{kg} / \mathrm{m}^{2}\right)$} \\
\hline Underweight $<18.5$ & 2.22 & 2.79 & 3.79 & 4.08 & 3.35 & 2674 \\
\hline Normal weight, $18.5-24.9$ & 46.83 & 46.02 & 43.31 & 39.31 & 37.18 & 53327 \\
\hline Overweight, 25-29.9 & 35.48 & 33.55 & 32.37 & 31.78 & 32.34 & 43915 \\
\hline $\begin{array}{l}\text { Moderately obese, } \\
\text { 30-34.9 }\end{array}$ & 11.72 & 12.44 & 13.15 & 15.62 & 16.57 & 17840 \\
\hline Severely obese, >34.9 & 3.75 & 5.2 & 7.38 & 9.22 & 10.57 & 7815 \\
\hline \multicolumn{7}{|l|}{ ADG score $†$} \\
\hline Mean (SD) & $\begin{array}{l}3.42 \\
(0.06)\end{array}$ & $\begin{array}{l}3.95 \\
(0.22)\end{array}$ & $\begin{array}{l}5.76 \\
(0.80)\end{array}$ & $\begin{array}{l}7.29 \\
(1.13)\end{array}$ & $\begin{array}{l}10.13 \\
(0.709)\end{array}$ & 129467 \\
\hline \multicolumn{7}{|l|}{ Physical activity status } \\
\hline Active & 32.24 & 24.09 & 18.2 & 16.94 & 14.95 & 35482 \\
\hline Moderate & 27.08 & 24.73 & 19.25 & 18.8 & 14.5 & 33791 \\
\hline Inactive & 40.65 & 51.14 & 62.22 & 64.2 & 70.55 & 60149 \\
\hline \multicolumn{7}{|l|}{ On-Marg deprivation } \\
\hline 1 (least marginalised) & 24.86 & 20.76 & 12.81 & 15.84 & 15.43 & 23405 \\
\hline 2 & 22.03 & 20.22 & 18.21 & 15.72 & 13.13 & 25427 \\
\hline 3 & 19.84 & 19.72 & 18.93 & 19.23 & 17.55 & 26391 \\
\hline 4 & 16.74 & 18.99 & 22.71 & 20.01 & 21.85 & 26386 \\
\hline 5 (most marginalised) & 15.57 & 19.29 & 26.2 & 28.04 & 31.52 & 26073 \\
\hline \multicolumn{7}{|l|}{ Education level } \\
\hline$<$ secondary & 4.62 & 6.52 & 9.87 & 12.4 & 12.91 & 12302 \\
\hline Secondary grad & 10.79 & 12.25 & 13.65 & 12.78 & 16.24 & 18047 \\
\hline > secondary & 79.93 & 75.6 & 69.26 & 68.55 & 61.28 & 94084 \\
\hline Unknown & 4.66 & 5.63 & 7.22 & 6.28 & 9.57 & 5034 \\
\hline \multicolumn{7}{|l|}{ Alcohol consumption } \\
\hline Heavy drinker & 7.94 & 8.62 & 8.08 & 9.11 & 11.6 & 11502 \\
\hline Moderate drinker & 24.62 & 20.19 & 15.43 & 12.57 & 7.58 & 17934 \\
\hline Light drinker & 15.37 & 13.61 & 11.42 & 9.26 & 7.44 & 28413 \\
\hline Never drinker & 50.79 & 56.33 & 63.66 & 67.55 & 71.93 & 69785 \\
\hline \multicolumn{7}{|l|}{ Smoking status } \\
\hline Current smoker & 17.38 & 22.71 & 31.65 & 36.52 & 43.75 & 30875 \\
\hline Former smoker & 22.92 & 20.53 & 17.48 & 19.76 & 19.43 & 32623 \\
\hline
\end{tabular}


Table 1 Continued

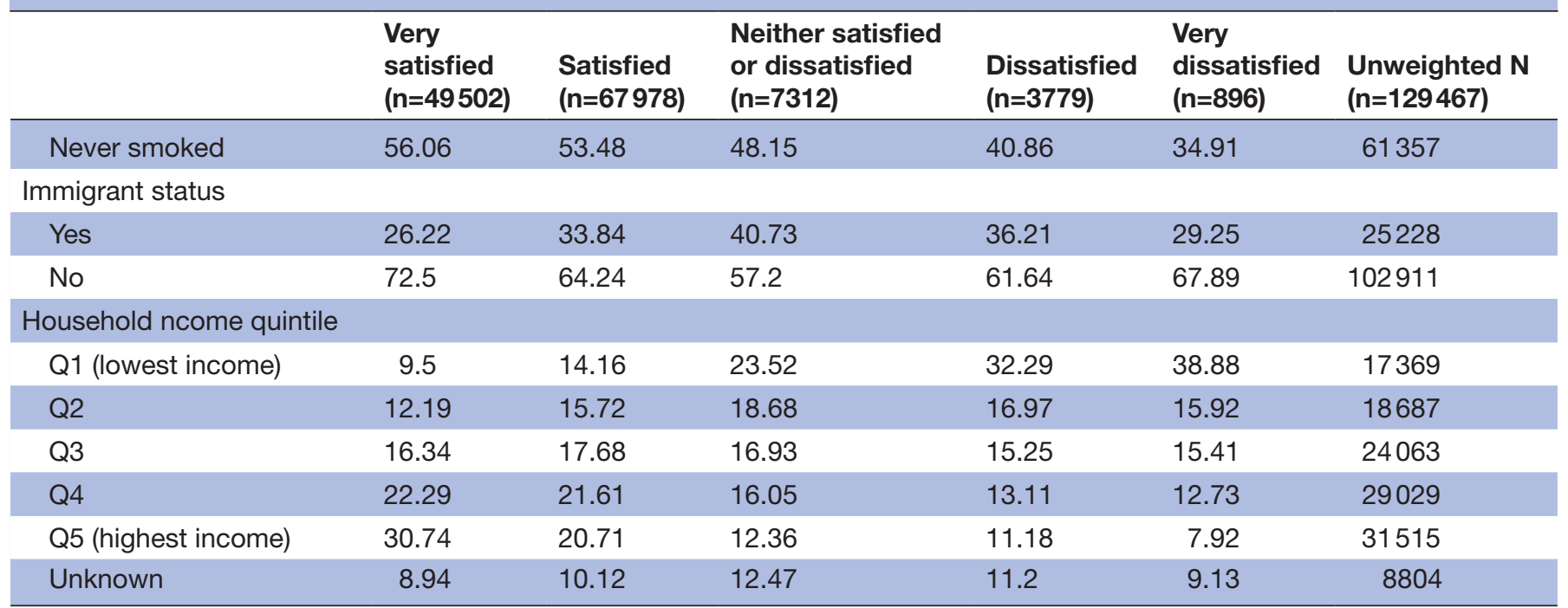

*Weighted through surveysampling weights provided by Statistics Canada.

†ADG score is a weighted score based on an individual's ADGs. This method has been validated elsewhere. ${ }^{26}$

ACSC, ambulatory care sensitive condition; ADG, aggregate diagnosis group; BMI, body mass index; ON-Marg, Ontario Marginalization Index.

To evaluate the proportional hazards assumption for life satisfaction, a plot of $\log (-\log$ (survival) ) versus the logarithm of follow-up time in days was run. With this method, the proportional hazards assumption is met if the plot produces parallel curves.

A joint-effects model was used to test if the relationship between life satisfaction and avoidable hospitalisations varied by SES. A joint-effects variable, which contains each combination of life satisfaction and household income, was included in the model while controlling for age, sex, smoking status, alcohol consumption, physical activity and BMI.

We ran sensitivity analyses by rerunning the fully adjusted model with a consistent survival time of 5 years. Additionally, models for individuals who did not have an event in the first 2 years of the study were run to control for undocumented comorbidity. The subdistribution hazards model, which was initially developed by Fine and Gray ${ }^{28}$ was run to test the possibility of death behaving as a competing risk.

Survey and bootstrap sampling weights provided by Statistics Canada were applied in all descriptive and survival regression analyses to account for the complex survey design and to maintain population representativeness. ${ }^{29}$ The bootstrap sampling weights were applied using balanced repeated replication, in order to properly calculate CIs. Finally, all statistical analyses were performed in 2018 and 2019 using SAS V.9.4.

\section{Patient involvement}

Patients were not involved in the development of the research question, outcome measures, recruitment, design or the implementation of the study objectives. Furthermore, no patients were consulted on the interpretation of results, and there are no plans to disseminate the results of this study to the relevant participants or their communities.

\section{RESULTS}

After combining the five cycles of data linked to the RPDB and excluding those less than 18, older than 74 , in multiple survey cycles, missing life satisfaction, had an ACSC-related hospitalisation in the 2 years before the start of the study resulted in 129467 individuals remaining. Those who experienced an ACSC-related hospitalisation 2 years before their survey interview date were removed to examine the impact of life satisfaction on future hospitalisations for an ACSC and reduce the possibility of reverse causality (figure 1). In the case of people in multiple survey cycles $(n=1589)$, we used the earliest survey response. Each respondent was followed for a maximum of 14 years or until the study end date, after which we determined that 3037 individuals had experienced an ACSC-related hospitalisation.

The distributions of baseline characteristics according to life satisfaction categories are shown in table 1 . Those with the lowest levels of life satisfaction (very dissatisfied) compared with the highest level of life satisfaction (very satisfied) had a lower proportion in the youngest (18-34 years) age group ( $14.5 \%$ vs $30.6 \%)$, more likely to have less than secondary education (12.9\% vs $4.62 \%)$, had a greater proportion in the lowest income quintile $(38.9 \%$ vs 9.5\%), higher comorbidity levels (ADG score 10.1 vs 3.42), and higher disease-related risk factors such as smoking $(43.8 \%$ vs $17.4 \%)$ and physical inactivity $(70.6 \%$ vs $40.7 \%$ ) (table 1$)$. 
Table 2 Weighted ${ }^{*}$ distributions of baseline characteristics according to hospitalisation for an ACSC $(n=129467)$

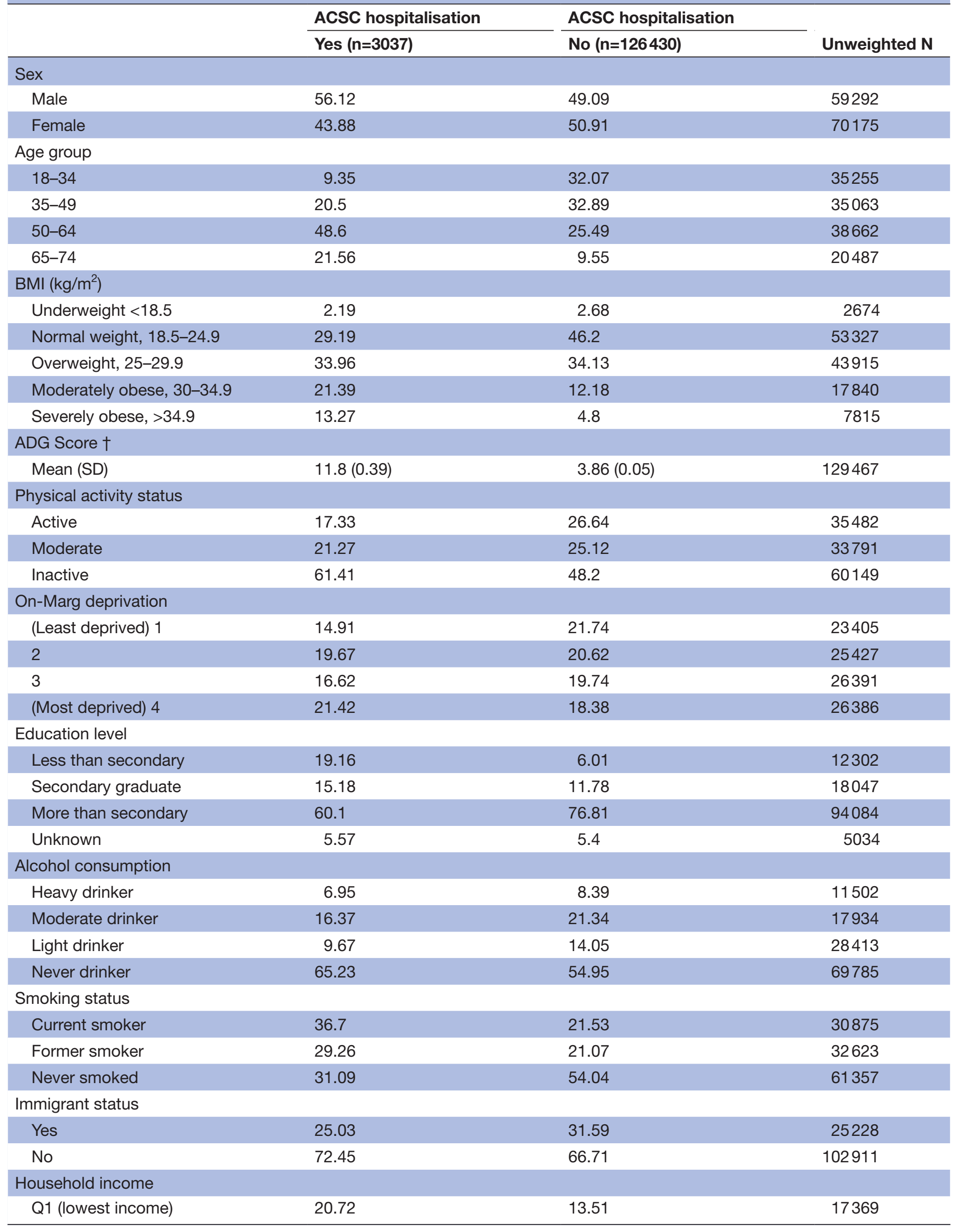




\begin{tabular}{|c|c|c|c|}
\hline & ACSC hospitalisation & ACSC hospitalisation & \\
\hline & Yes $(n=3037)$ & No $(n=126430)$ & Unweighted $\mathbf{N}$ \\
\hline Q2 & 16.09 & 14.61 & 18687 \\
\hline Q4 & 17.57 & 21.31 & 29029 \\
\hline Q5 (highest income) & 18.58 & 23.65 & 31515 \\
\hline
\end{tabular}

*Weighted through survey sampling weights provided by Statistics Canada.

†ADG score is a weighted score based on an individual's ADGs. This method has been validated elsewhere. ${ }^{26}$

ACSC, ambulatory care sensitive condition; ADG, aggregate diagnosis group; BMI, body mass index; ON-Marg, Ontario Marginalization Index.

The distributions of all the cohort characteristics according to ACSC status are show in table 2. Those that had an ACSC during the follow-up compared with those that did not were more likely to be in the older age group (65-74 years) $(21.6 \%$ vs $9.55 \%)$, more likely to have less than secondary education $(19.2 \%$ vs $6.01 \%)$, had a greater proportion in the lowest income quintile $(20.7 \%$ vs $13.5 \%)$, higher comorbidity levels (ADG score 11.8 vs 3.86) and higher disease-related risk factors such as smoking (36.7\% vs $21.5 \%$ ) and physical inactivity (61.4\% vs $48.2 \%$ ) (table 2 ).

Life satisfaction had a strong unadjusted relationship with hospitalisation for ACSC (unadjusted Kaplan-Meier curves are shown in online supplementary figure 1). Table 3 presents the unadjusted and adjusted HRs for the relationship between life satisfaction and ACSC-related hospitalisations, which include models that adjusted for age and sex, and then further adjusted for socioeconomic and lifestyle factors. Although full adjustment does reduce the size of the effect, the HR of an individual with the lowest levels of life satisfaction (dissatisfied and very dissatisfied combined) compared with those who were very satisfied is 2.71 (95\% CI 1.87 to 3.93 ). The observed relationship follows a dose-response pattern, or in other words, the HRs increase in size for each decreasing level of life satisfaction. For example, in the fully adjusted model, the HR for the middle life satisfaction category (neither satisfied nor dissatisfied) was 1.71 (95\% CI 1.36 to 2.14) while the satisfied category produced an HR of 1.32 (95\% CI 1.15 to 1.50$)$. Furthermore, the additional analyses (table 4) that controlled for ADG score, mood disease and anxiety separately did not substantially reduce the observed effect sizes with the added adjustment of ADG score having the largest impact (HR of 2.42, 95\% CI of 1.68 to 3.51 ). Finally, as the plot of survival by time according to life satisfaction produced parallel curves, the proportional hazards assumption was satisfied.

Regarding the joint effects model (table 5), individuals who identified as having both low life satisfaction and low household income produced a fully adjusted HR of 3.80 (95\% CI 2.13 to 6.73). Therefore, in comparison to the fully adjusted model presented in table 3 (HR of 2.71), poor SES individuals are at an increased risk of being hospitalised when reporting low life satisfaction.

A range of sensitivity analyses were conducted to test the robustness of the study findings. Both the 5-year survival model (where everyone was limited to a 5 -year follow-up) and the model which excluded individuals who had an event within the first 2 years did not change the effect sizes to a significant degree. The HRs for the lowest levels of life satisfaction compared with those who were very satisfied were 2.74 and 2.77 , respectively (online supplementary table 2). In the competing risk analysis (online supplementary table 3), the unadjusted HRs produced in the subdistribution hazard model were similar to those produced in the final model (4.38 vs 4.51 , respectively).

\section{DISCUSSION}

This study focuses on how life satisfaction can impact health system indicators such as avoidable hospitalisations in a general population cohort without a recent ACSC. We investigated this relationship and accounted for a wide variety of sociodemographic and behavioural risk factors. We saw a robust association that poor life satisfaction had a strong independent relationship with future ACSC hospitalisations. The lowest levels of life satisfaction (dissatisfied and very dissatisfied) being associated with almost a three times higher hazard of an avoidable hospitalisation compared with those who were very satisfied after accounting for several sociodemographic and behavioural confounders.

Previous studies have linked life satisfaction and related exposures (eg, positive affect or happiness) to health outcomes such as stroke ${ }^{11}$ and heart disease. ${ }^{10}$ Life satisfaction has also been shown to be associated with a wide variety of health behaviours. ${ }^{31}{ }^{31}$ For instance, one study found that those who exercised more were generally happier. ${ }^{32}$ Furthermore, life satisfaction has also been shown to be experienced differently across categories of SES. ${ }^{33}$ Due to the detailed survey, variables available from the survey data and the linkage of these data to complete hospitalisation outcomes from health administrative databases were able to adjust for these health behaviours and measures of SES 


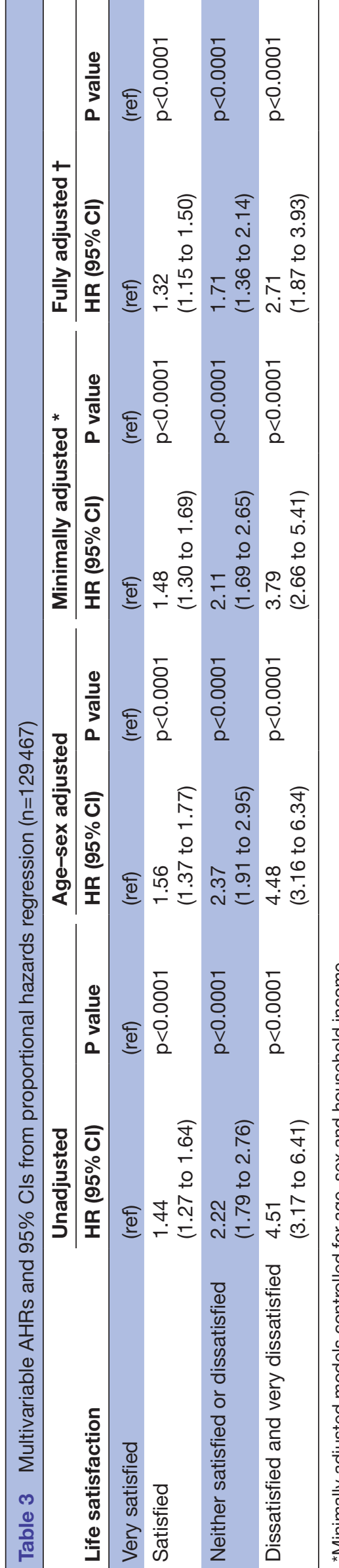

in our analysis as well as examine how the influence of life satisfaction is strengthen or lessened across levels of SES.

This study addresses an important gap in the literature by providing a robust population sample size and examining how life satisfaction related to a meaningful health system outcome. There are few studies that addresses life satisfaction or other forms of subjective well-being and their relationship to hospitalisations for ACSCs. Furthermore, the existing research is limited by small sample sizes and limited follow-up times to capture the relatively rare ACSC event. This study also has more direct implications for the health system given ACSCs are defined as conditions for which hospitalisations should be prevented, given timely and effective access to primary care. ${ }^{7}$ Considering the preventable nature of these conditions, hospitalisations for ACSCs are an ineffective use of healthcare resources and insight into the risk factors for these conditions can help improve health system functioning.

Unlike previous studies, we measured baseline life satisfaction on a sample who did not have an ACSC hospitalisation in the 2years prior while also presenting analyses that additionally controlled for comorbidity. These aspects of the study help mitigate the possibility that that poor life satisfaction could have been the result of the bidirectional relationship between poor health and life satisfaction. A possible explanation for the observed results is that individuals who experience poor life satisfaction tend to have higher rates of depression, given its observed relationship with poor health outcomes. ${ }^{34}{ }^{35}$ To address this, we further adjusted for mood disease and found that this has little effect on attenuated the observed association. The survey did not include continuous measures that could capture subthreshold levels or undiagnosed anxiety or depression.

\section{Limitations}

We acknowledge that this study has some limitations and interpretive cautions. First, this study was an observational study and although we controlled for several potential confounders and excluded those with a recent history of ACSC at baseline through linkage, we cannot rule out the possibility of unmeasured or residual confounding. We note, however, that the effect sizes are large, and this study did control for many more confounders than previous studies through a combination of survey data in addition to health administrative variables to capture comorbidity. Second, we measured life satisfaction at one point in time (survey interview date). Research on life satisfaction has shown it to be consistent over time ${ }^{36}$; however, it is possible that life satisfaction could have changed during the study period. Therefore, we only capture the effect from that initial time point and cannot account for the influence of changes in life satisfaction that happen following the baseline assessment. Furthermore, there are other instruments that can be used to assess life satisfaction that were not available in this survey. Finally, life satisfaction is a subjective measure. It has been shown to be an accurate and robust measure, it is still up to the individuals to judge and reflect on their life satisfaction. This means that its meaning can 
Table 4 Multivariable AHRs and 95\% Cls from proportional hazards regression after additionally adjusting for ADG score, mood disease and anxiety ( $n=129467)$

\begin{tabular}{|c|c|c|c|c|c|c|}
\hline & \multicolumn{2}{|c|}{ Fully adjusted* with ADG score } & \multicolumn{2}{|c|}{ Fully adjusted* with mood disease } & \multicolumn{2}{|c|}{ Fully adjusted ${ }^{*}$ with anxiety } \\
\hline & HR $(95 \% \mathrm{Cl})$ & $P$ value & HR $(95 \% \mathrm{Cl})$ & $P$ value & HR (95\% Cl) & $P$ value \\
\hline \multicolumn{7}{|l|}{ Life satisfaction } \\
\hline Very satisfied & (ref) & (ref) & (ref) & (ref) & (ref) & (ref) \\
\hline Satisfied & 1.28 (1.12 to 1.46$)$ & 0.0004 & 1.30 (1.13 to 1.50$)$ & 0.0002 & 1.31 (1.14 to 1.49$)$ & 0.0001 \\
\hline $\begin{array}{l}\text { Neither satisfied } \\
\text { or dissatisfied }\end{array}$ & 1.56 (1.25 to 1.96$)$ & 0.0001 & 1.62 (1.29 to 2.05$)$ & $p<0.0001$ & 1.65 (1.31 to 2.08$)$ & $\mathrm{p}<0.0001$ \\
\hline $\begin{array}{l}\text { Dissatisfied and } \\
\text { very dissatisfied }\end{array}$ & 2.42 (1.68 to 3.51$)$ & $p<0.0001$ & 2.45 (1.68 to 3.55$)$ & $p<0.0001$ & 2.52 (1.76 to 3.61$)$ & $\mathrm{p}<0.0001$ \\
\hline
\end{tabular}

*Models controlled for age, sex, household income, physical activity, BMI, alcohol consumption, smoking status in addition to the variable specified.

ADG, aggregate diagnosis group; AHRs, Adjusted Hazard Ratios; BMI, body mass index.

differ based on the individual, which can result in variation in the exposure. Despite these limitations, this study has still provided an essential contribution to the literature by being one of the first assessments of life satisfaction on avoidable hospitalisation that uses a longitudinal population-based study design while measuring this exposure before the hospitalisation outcome.

\section{Mechanisms}

This study did not directly address mechanisms by which life satisfaction can influence avoidable hospitalisations; however, these have been studied by others. Out of the existing literature, there are three main mechanisms that could help explain the observed relationship: behavioural, health service use and biological. Subjective well-being has been shown to impact a variety of health behaviours, such as increased physical activity ${ }^{37-40}$ and reduced smoking habits. ${ }^{40}$ However, many of these studies lacked proper adjustment of confounding variables such as SES and psychological distress. ${ }^{40}$ Regarding health service use, a study from 2014 found that participants who were identified as having a greater purpose in life were more likely to receive preventative health services such as mammograms or colonoscopies. ${ }^{41}$ What remains to be seen regarding this mechanism is whether the use of these services reflects access to primary care, or the decisions made by the individuals themselves to seek these services. However, a recent population-based cohort study noted how hospitalisations for ACSCs could not be explained by a lack of access to primary care, ${ }^{42}$ and therefore, it is possible that this phenomenon is a result of individual decisions that are influenced by their satisfaction with life. Finally, biological mechanisms could also play a contributing role. A recent meta-analysis noted how individuals who were identified as having greater psychological well-being experienced favourable lipid profiles. ${ }^{43}$ However, these associations were largely mitigated once behavioural characteristics were taken into account. ${ }^{43}$ Our study did not compare the extent to which life satisfaction is associated with ACSC compared with other types of hospitalisation. This was out of scope for this current study, but in a future analysis could help provide insight into mechanisms that are specific to preventable hospitalisations. Our study did not examine

Table 5 Multivariable AHRs and 95\% Cls from joint effects models ( $n=129467)$

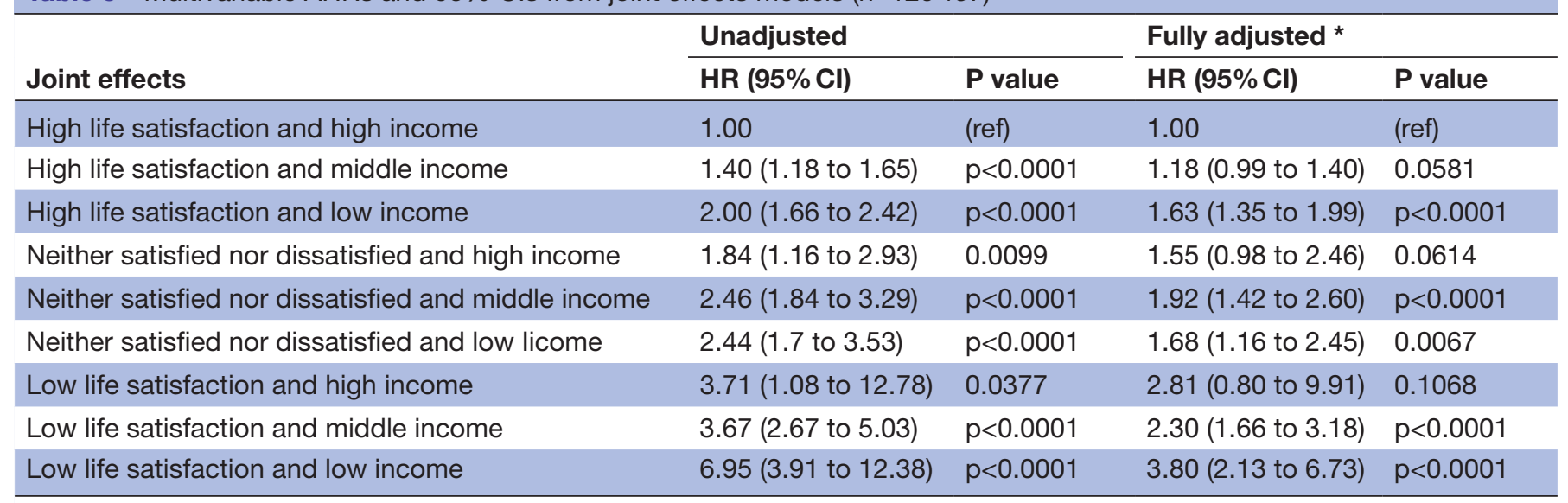

*Models controlled for age, sex, household income, physical activity, BMI, alcohol consumption, and, smoking status.

AHR, Adjusted Hazard Ratios. 
whether ACSC hospitalisations were reduced for Ontario hospitals, but instead focused on individual's risk based on their life satisfaction measure. In future studies, analyses at the system level (ie, ACSC hospitalisations in Ontario hospitals) would supplement these findings to better understand the health system impact.

\section{Conclusions}

This study demonstrates that life satisfaction is associated with hospitalisations for ACSCs, even after controlling for socioeconomic characteristics, health behaviours, comorbidities and mental health factors. Furthermore, more socioeconomically deprived individuals were shown to be at an increased risk. While governments plan to improve the sustainability and functioning of their health systems, there is a greater need to understand social supports that can improve life satisfaction costly and preventable conditions such as ACSCs. The findings of this study suggest that broader considerations, such as life satisfaction, can potentially influence avoidable hospitalisations, a burden to individuals and healthcare systems. Further research in this area may contribute to the development of wide-ranging approaches to target a potentially avoidable burden on the health system.

\section{Twitter Laura C Rosella @LauraCRosella}

Contributors LCR and VG conceptualised the study, TW created the database and EDP planned the analysis, analysed the data and drafted and revised the paper. LCR is the guarantor. All authors contributed to the manuscript and revised the draft paper. All have approved the final version for publication.

Funding This research was supported by a Canada Research Chair held by LCR (CRC-950-23072). This study was supported by ICES, which is funded by an annual grant from the Ontario Ministry of Health and Long-Term Care.

Disclaimer The opinions, results and conclusions reported in this paper are those of the authors and are independent from the funding sources.

Competing interests None declared.

Patient consent for publication Not required.

Ethics approval This project received ethics approval from the Health Sciences Research Ethics Board at the University of Toronto (Ref \# 36123, 15 August 2018).

Provenance and peer review Not commissioned; externally peer reviewed.

Data availability statement Data may be obtained from a third party and are not publicly available. The data set from this study is held securely in coded form at ICES. While data sharing agreements prohibit ICES from making the data set publicly available, access may be granted to those who meet prespecified criteria for confidential access (available at www.ices.on.ca/DAS). The full data set creation plan and underlying analytical code are available from the authors on request, understanding that the programmes may rely on coding templates or macros that are unique to ICES.

Open access This is an open access article distributed in accordance with the Creative Commons Attribution Non Commercial (CC BY-NC 4.0) license, which permits others to distribute, remix, adapt, build upon this work non-commercially, and license their derivative works on different terms, provided the original work is properly cited, appropriate credit is given, any changes made indicated, and the use is non-commercial. See: http://creativecommons.org/licenses/by-nc/4.0/.

ORCID iD

Laura C Rosella http://orcid.org/0000-0003-4867-869X

\section{REFERENCES}

1 Zaninotto P, Wardle J, Steptoe A. Sustained enjoyment of life and mortality at older ages: analysis of the English longitudinal study of ageing. BMJ 2016;355:i6267.
2 Boehm JK, Trudel-Fitzgerald C, Kivimaki M, et al. The prospective association between positive psychological well-being and diabetes. Health Psychol 2015;34:1013-21.

3 Sabatini F. The relationship between Happiness and health: evidence from Italy. Soc Sci Med 2014;114:178-87.

4 Carrière GM, Kumar MB, Sanmartin C. Hospitalization for ambulatory care sensitive conditions among urban Métis adults. Health Rep 2017;28:3-11.

5 Caminal J, Starfield B, Sánchez E, et al. The role of primary care in preventing ambulatory care sensitive conditions. Eur J Public Health 2004;14:246-51.

6 Brown AD, Goldacre MJ, Hicks N, et al. Hospitalization for ambulatory care-sensitive conditions: a method for comparative access and quality studies using routinely collected statistics. Can J Public Health 2001;92:155-9.

7 Billings J, Teicholz N. Uninsured patients in district of Columbia hospitals. Health Aff 1990;9:158-65.

8 Magan P, Otero A, Alberquilla A, et al. Geographic variations in avoidable hospitalizations in the elderly, in a health system with universal coverage. BMC Health Serv Res 2008;8:42.

9 Tomkins S. Affect imagery consciousness: the positive affects. New York: Springer Publishing Inc, 1963.

10 Davidson KW, Mostofsky E, Whang W. Don't worry, be happy: positive affect and reduced 10-year incident coronary heart disease: the Canadian Nova Scotia Health Survey. Eur Heart $J$ 2010;31:1065-70.

11 Feller S, Teucher B, Kaaks R, et al. Life satisfaction and risk of chronic diseases in the European prospective investigation into cancer and nutrition (EPIC)-Germany study. PLoS One 2013;8:e73462

12 Grant N, Wardle J, Steptoe A. The relationship between life satisfaction and health behavior: a cross-cultural analysis of young adults. Int J Behav Med 2009;16:259-68.

13 Goel V, Rosella LC, Fu L, et al. The relationship between life satisfaction and healthcare utilization: a longitudinal study. Am J Prev Med 2018;55:142-50.

14 Audulv Åsa, Ghahari S, Kephart G, et al. The taxonomy of everyday self-management strategies (TEDSS): a framework derived from the literature and refined using empirical data. Patient Educ Couns 2019;102:367-75.

15 Schulman-Green D, Jaser SS, Park C, et al. A metasynthesis of factors affecting self-management of chronic illness. J Adv Nurs 2016;72:1469-89.

16 Liddy C, Blazkho V, Mill K. Challenges of self-management when living with multiple chronic conditions: systematic review of the qualitative literature. Can Fam Physician 2014;60:1123-33.

17 Konstam V, Salem D, Pouleur H, et al. Baseline quality of life as a predictor of mortality and hospitalization in 5,025 patients with congestive heart failure. Am J Cardiol 1996;78:890-5.

18 González-Freire B, Vázquez-Rodríguez I, Marcos-Velázquez P, et al. Repression and coping styles in asthmatic patients. J Clin Psychol Med Settings 2010;17:220-9.

19 Scheier MF, Matthews KA, Owens JF, et al. Optimism and rehospitalization after coronary artery bypass graft surgery. Arch Intern Med 1999;159:829-35.

20 Ronaldson A, Molloy GJ, Wikman A, et al. Optimism and recovery after acute coronary syndrome: a clinical cohort study. Psychosom Med 2015;77:311-8.

21 Sanmartin CA, Khan S, Statistics Canada, Canadian Electronic Library (Firm). Hospitalizations for ambulatory care sensitive conditions (ACSC) the factors that matter. Ottawa, Ontario: Statistics Canada, 2011. http://myaccess.library.utoronto.ca/login?url=http:// books.scholarsportal.info/viewdoc.html?id=/ebooks/ebooks1/ gibson_chrc/2011-10-10/1/10490988

22 Statistics Canada. Canadian community health Survey- annual component (CCHS), 2016. Available: http://www23.statcan.gc.ca/ imdb/p2SV.pl?Function=getSurvey\&ld=259374

23 Statistics Canada. Canadian community health survey, 2003: user guide for the public use of Microdata file. Ottawa, 2005.

24 Canadian Institute for Health Information. Indicator library: ambulatory care sensitive conditions. Available: http:// indicatorlibrary.cihi.ca/display/HSPIL/Ambulatory+Care+Sensitive+ Conditions

25 Weiner JP, Abrams C, Bodycombe D. The Johns Hopkins ACG case-mix system version 6.0 release notes. Baltimore: Johns Hopkins Bloomberg School of Public Health, 2003.

26 Austin PC, van Walraven C, Wodchis WP, et al. Using the Johns Hopkins aggregated diagnosis groups (ADGS) to predict mortality in a general adult population cohort in Ontario, Canada. Med Care 2011:49:932-9 
27 Matheson FI, Dunn JR, Smith KLW, et al. Development of the Canadian marginalization index: a new tool for the study of inequality. Can J Public Health 2012;103:S12-16.

28 Fine JP, Gray RJ. A proportional hazards model for the Subdistribution of a competing risk. J Am Stat Assoc 1999;94:496-509.

29 Kolenikov S. Resampling variance estimation for complex survey data. Stata J 2010;10:165-99.

30 Lyubomirsky S, King L, Diener E. The benefits of frequent positive affect: does Happiness lead to success? Psychol Bull 2005;131:803-55.

31 Pressman SD, Cohen S. Does positive affect influence health? Psychol Bull 2005;131:925-71.

32 Lathia N, Sandstrom GM, Mascolo C, et al. Happier people live more active lives: using Smartphones to link Happiness and physical activity. PLoS One 2017;12:e0160589.

33 Barger SD, Donoho CJ, Wayment HA. The relative contributions of race/ethnicity, socioeconomic status, health, and social relationships to life satisfaction in the United States. Qual Life Res 2009;18:179-89.

34 Cuijpers P, de Graaf R, van Dorsselaer S. Minor depression: risk profiles, functional disability, health care use and risk of developing major depression. J Affect Disord 2004;79:71-9.

35 Black SA, Markides KS, Ray LA. Depression predicts increased incidence of adverse health outcomes in older Mexican Americans with type 2 diabetes. Diabetes Care 2003;26:2822-8.
36 Bonikowska A, Helliwell JF, Hou F, et al. An assessment of life satisfaction responses on recent statistics Canada surveys. Soc Indic Res 2014;118:617-43.

37 Baruth M, Lee D-C, Sui X, et al. Emotional outlook on life predicts increases in physical activity among initially inactive men. Health Educ Behav 2011;38:150-8.

38 Kim ES, Kubzansky LD, Soo J, et al. Maintaining healthy behavior: a prospective study of psychological well-being and physical activity. Ann Behav Med 2017;51:337-47.

39 Sin NL, Moskowitz JT, Whooley MA. Positive affect and health behaviors across 5 years in patients with coronary heart disease: the heart and soul study. Psychosom Med 2015;77:1058-66.

40 Boehm JK, Chen Y, Koga H, et al. Is optimism associated with healthier Cardiovascular-Related behavior? Circ Res 2018;122:1119-34

41 Kim ES, Strecher VJ, Ryff CD. Purpose in life and use of preventive health care services. Proc Natl Acad Sci U S A 2014;111:16331-6.

42 Vuik SI, Fontana G, Mayer E, et al. Do hospitalisations for ambulatory care sensitive conditions reflect low access to primary care? an observational cohort study of primary care usage prior to hospitalisation. BMJ Open 2017;7:e015704.

43 Soo J, Kubzansky LD, Chen Y, et al. Psychological well-being and restorative biological processes: HDL-C in older English adults. Soc Sci Med 2018;209:59-66. 